\title{
A kilometre-range distributed relative humidity sensor
}

Tiago F. P. Neves, Li Zhang, Fan Yang, Kenny H. Tow, Paolo Petagna, et al.

Tiago F. P. Neves, Li Zhang, Fan Yang, Kenny H. Tow, Paolo Petagna, Luc Thévenaz, "A kilometre-range distributed relative humidity sensor," Proc. SPIE 11199, Seventh European Workshop on Optical Fibre Sensors, 1119922 (28 August 2019); doi: 10.1117/12.2540007

SPIE Event: Seventh European Workshop on Optical Fibre Sensors, 2019, Limassol, Cyprus 


\title{
A Kilometre-Range Distributed Relative Humidity Sensor
}

\author{
Tiago F. P. Neves *ab, Li Zhang ${ }^{\mathrm{b}}$, Fan Yang ${ }^{\mathrm{b}}$, Kenny Hey Tow ${ }^{\mathrm{c}}$, Paolo Petagna ${ }^{\mathrm{a}}$, Luc Thévenaz ${ }^{\mathrm{b}}$ \\ ${ }^{a}$ Experimental Department, Detector Technology Group, CERN - European Organization for

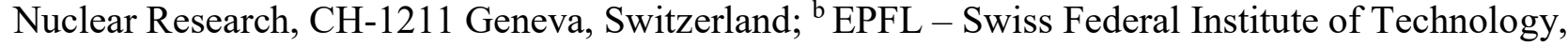 \\ Group for Fibre Optics, SCI STI LT, Station 11, 1015 Lausanne, Switzerland; ' RISE Acreo, \\ Department of Fiber Optics, Electrum 236, 164 40, Kista, Sweden
}

\begin{abstract}
Fibre optics sensors have been identified as very good candidates for environmental monitoring inside the silicon detectors operated at CERN's Large Hadron Collider. In this study, we present the results from the first highly sensitive relative humidity distributed sensor with kilometres sensing range. The setup is a $70 \mathrm{~cm}$ spatial resolution phase-sensitive Optical Time Domain Reflectometry (OTDR) and is able to monitor fibre lengths up to $10 \mathrm{~km}$. The coating effect is also evaluated, analysing different coating thicknesses, number of coating layers, different manufacturing and different materials. Relative humidity tests were performed at two different temperatures $\left(25^{\circ} \mathrm{C}\right.$ and $\left.42^{\circ} \mathrm{C}\right)$. Polyimide coated fibres show in general a higher humidity sensitivity then a standard acrylate coated fibre, while acrylate fibres offer the fastest response and settling time. The system is able to resolve $0.1 \% \mathrm{RH}$ and all tested fibres proved to be good candidates to be employed in a distributed relative humidity sensor. If the requirements are a fast time response and short settling time at room temperature, the standard acrylate coated fibres are the best candidates. However, if the requirements are high sensitivity and measurement stability at different temperatures, the polyimide-coated fibres offer advantages on several aspects.
\end{abstract}

Keywords: Humidity, fibre, temperature, sensing, continuous, distributed, phase-OTDR, coating, polyimide, acrylate, Rayleigh, CERN

\section{INTRODUCTION}

Silicon detectors operated at CERN's (European Organization for Nuclear Research) LHC (Large Hadron Collider) are subject to high radiations, which can rapidly downgrade their performance unless they are constantly kept at negative temperatures (typically $<-30^{\circ} \mathrm{C}$ ). Therefore, the relative humidity $(\mathrm{RH})$ inside the detector enclosure should be strictly controlled in order to avoid any risk of condensation, potentially damaging the associated complex electronics. Any environmental sensor to be installed in a silicon detector at LHC should ideally fulfil the requirements of being radiation resistant and insensitive to magnetic fields, while having small dimensions, reliable reading across long distances, and ease of multiplexing to form large network of sensors. Taking into account these requirements, fibre optics sensors (FOS) have been identified as very good candidates, in particular for their good withstanding under strong irradiation [1]. Different techniques are available to produce FOS sensitive to relative humidity (RH-FOS), finding applications in several fields, as reported by a recent review [2]. At CERN a first generation of RH-FOS, based on polyimide coated Fiber Bragg Gratings (FBG) [3] was originally developed and installed in a network of 72 optic fibre-based thermo-hygrometers in the CMS experiment [4]. A second generation of RH-FOS, based on Long Period Gratings (LPG) with titanium dioxide $\left(\mathrm{TiO}_{2}\right)$ coating was later developed ( [5] [6] ), in particular targeting very high sensitivity in the low humidity range $(\mathrm{RH}<5 \%)$. However, RH-FOS based on gratings are only sensitive at the locations where the gratings are present, and also have intrinsic limits in terms of multiplexing capabilities (particularly severe for the LPG-based ones). For these reasons, a third generation of RH-FOS is being studied, based on the distributed sensing technology, which enables to turn the whole fibre into a continuous linear sensor [7]. The concept of fully distributed RH-FOS has been recently demonstrated by Thomas and Hellevang [8], who described a 64-meters long distributed RH-FOS with centimetre spatial resolution, with a commercial polyimide coating as the sensing element. However, Optical Frequency Domain Reflectometry (OFDR) was used to measure Rayleigh scattering in the fibre: this technique is limited in its sensing range, restricting measurements over only few tens of meters. In this work, we present results from the first highly sensitive relative humidity distributed sensor up to 10 kilometre sensing range, based on a $70 \mathrm{~cm}$ spatial resolution phase-sensitive Optical Time Domain Reflectometry ( $\phi$-OTDR). This configuration is highly compatible for a systematic study of different coatings under identical conditions by appending different fibre segments and interrogating them simultaneously in a single acquisition.

*tiago.neves@cern.ch; phone +41 75411 2511; cern.ch

Seventh European Workshop on Optical Fibre Sensors, edited by Kyriacos Kalli, Gilberto Brambilla, Sinead O'Keeffe Proc. of SPIE Vol. 11199, 1119922 - (c) 2019 SPIE · CCC code: 0277-786X/19/\$21 · doi: 10.1117/12.2540007 


\section{SENSING PRINCIPLES}

The $\phi$-OTDR interrogation technique measures the noise-like but static Rayleigh backscattering signal caused by the frozen and random longitudinal entropic fluctuations of the refractive index (RI) alongside the optical fibre. A deterministic spectral shift of these random signal variations is caused by the stress induced $(\varepsilon)$ by the deformed coating in presence of water molecules. The humidity causes an expansion of the coating, stressing the fibre and, if the fibre is totally free of static mechanical strain, the total backscattered signal is defined as [9]:

$$
\Delta \varepsilon_{\text {total }}=\left(1-P_{e}\right) \varepsilon+\left[\left(1-P_{e}\right) \alpha+\xi\right] \Delta T
$$

where $P_{e}$ is the photo-elastic coefficient of the optical fiber, $\alpha$ and $\xi$ are the thermal expansion and thermal optic coefficients respectively and $T$ the temperature. Non-homogeneities in the RI scatter the incident light and induce a phase change:

$$
\phi=\int_{0}^{z} \beta d x=2 \pi \frac{v}{c} \int_{0}^{z} n_{e f f}(x) d x
$$

where $\beta$ is the propagation constant, $v$ is the optical frequency, $c$ the vacuum speed of the light and $n_{\text {eff }}$ the effective refractive index. In a $\phi$-OTDR sensor, the Rayleigh intensity traces are repeatedly acquired using different optical frequencies and the scanning range determines the maximum detectable temperature or strain change. The data analysis procedure requires the use of a reference trace, which is then compared in the frequency domain with the actual Rayleigh measurement. The maximum of similarity corresponds to the frequency shift induced by temperature or strain changes at a given position. A detailed description of the principle and of the experimental setup used in this study is provided in [10].

\section{EXPERIMENTAL SETUP}

A distributed feedback laser with $1 \mathrm{MHz}$ linewidth is employed as a laser source. Then, a SOA (Semiconductor Optical Amplifier) driven by a pulse generator (PG) is used to shape the continuous laser light into a coherent pulse. After the pulse shaping, an Erbium-doped fibre amplifier (EDFA) is used to amplify the pulse before launching it to the fibre under test through a circulator. The output of the circulator is connected to another EDFA, which amplifies the Rayleigh backscattered signal and then, a tuneable filter $(1550 \mathrm{~nm})$ is connected to filter out the EDFA amplified spontaneous emission before photo-detection. The photodetector is then connected to a fast oscilloscope for data acquisition. A LabVIEW VI controls the laser current swapping and acquires all the traces measured by the oscilloscope. The setup is completed by 12 different fibres installed in stress free spools inside a Climatic Chamber (Binder 720L). Reference temperature (PT100) and RH (HIH4000) sensors are installed separately in all the fibre spools.

\section{RESULTS}

Relative humidity tests were performed at two different temperatures $\left(25^{\circ} \mathrm{C}\right.$ and $\left.42^{\circ} \mathrm{C}\right)$ and the presented results are corrected for temperature fluctuations. The temperature reference is provided by a 10 meters bare fibre (stripped coating), which is only temperature-sensitive. Figure 1A shows that the compensation method is valid, proving the matching responses between reference sensor (in black) and bare fibre (in blue) and the total immunity of the response to humidity.
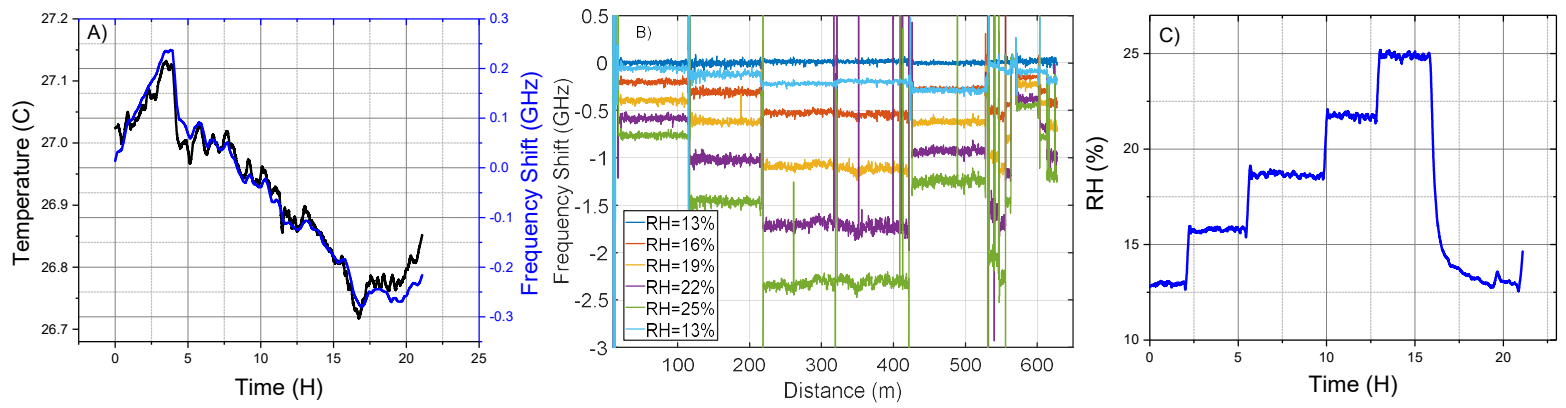

Figure 1. A) Temperature reference method. B) $\phi$-sensitive OTDR trace at different RH levels at $25^{\circ} \mathrm{C}$. C) $\mathrm{RH}$ variation.

From the full OTDR trace shown in Fig. 1B), one can conclude that different fibres show different sensitivities due to the different coatings thicknesses and materials. To compare the sensitivities and the response and stability times, an analysis of the temporal frequency shift variation of the fibres has been separately performed. 

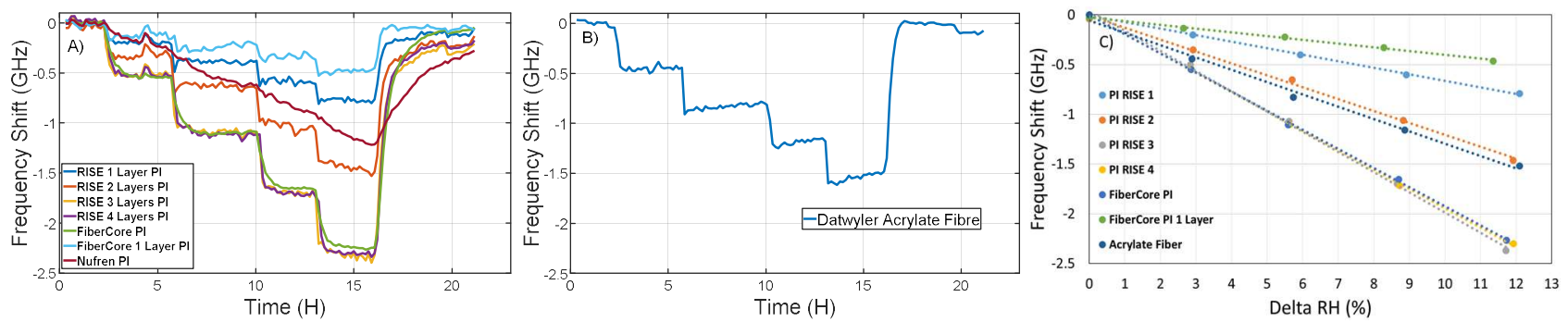

Figure 2. A) grouped plot of all different Polyimide (PI) fibres time responses. B) Response of the Acrylate (AC) fibre. C) Calibration fitting of all fibres.

Analysing Figure 2A and 2B, the RH sensitivity of the fibres can be calculated and using these linear sensitivities, Figure $2 \mathrm{C}$, it is possible to reconstruct the RH from the fibres frequency shift. Figure 3 shows, in black, the reconstructions of the relative humidity variation measured by three different fibres and, in blue, the relative humidity variation from the analog sensors. The same relative humidity test was carried out at $42^{\circ} \mathrm{C}$ and the results are summarised in Table 1 .
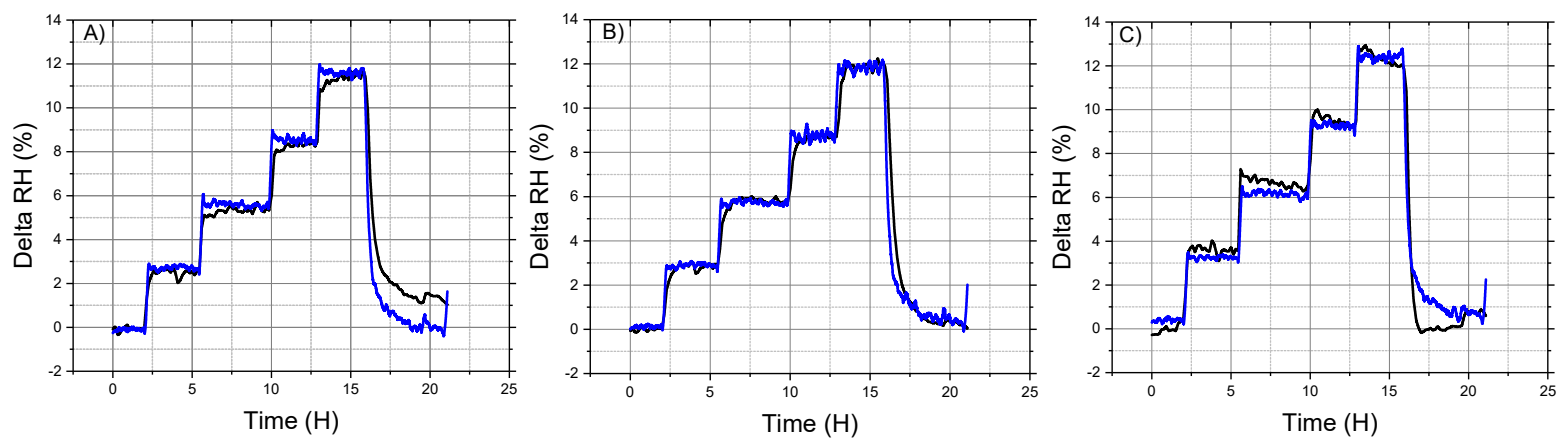

Figure 3. In blue, the analog references and in black the RHs reconstructed from the fibres frequency shift. A) RH from RISE Fibre with 3 coating layers. B) RH from FiberCore commercial fibre. C) RH from Acrylate-coated fibre.

Table 1. Relative Humidity sensitivities at different temperatures. Results in $\mathrm{MHz} / \% \mathrm{RH}$.

\begin{tabular}{|c|c|c|c|c|c|c|c|c|c|}
\hline Temp. & Rise 1 & Rise 2 & Rise 3 & Rise 4 & Nufren & FiberCore 1 & FiberCore 2 & FiberCore 3 & Acrylate \\
\hline $25^{\circ} \mathrm{C}$ & 65.6 & 119.6 & 200.3 & 193.4 & -- & 172.5 & 192.3 & 37.8 & 124.1 \\
\hline $42^{\circ} \mathrm{C}$ & 59.6 & 129.8 & 197.3 & 169.4 & 83.9 & 161.7 & 178.7 & 36.4 & 56.8 \\
\hline
\end{tabular}

Finally, a piece of 100 meters of Polyimide fibre (RISE 1 Layer) was connected to a 10 kilometres fibre spool, this latter being placed outside the climatic chamber and a simple one-step RH test was carried out on the test sample. The long fibre was placed outside the chamber purposely to make it subject to conditions different to the fibre test sample. Figure 4 shows that the system is still able to detect this RH variation without significant penalty after 10-kilometre length, showing the same expected sensitivity. The random variations are due to the uncontrolled environment of the 10 kilometres fibre spool.
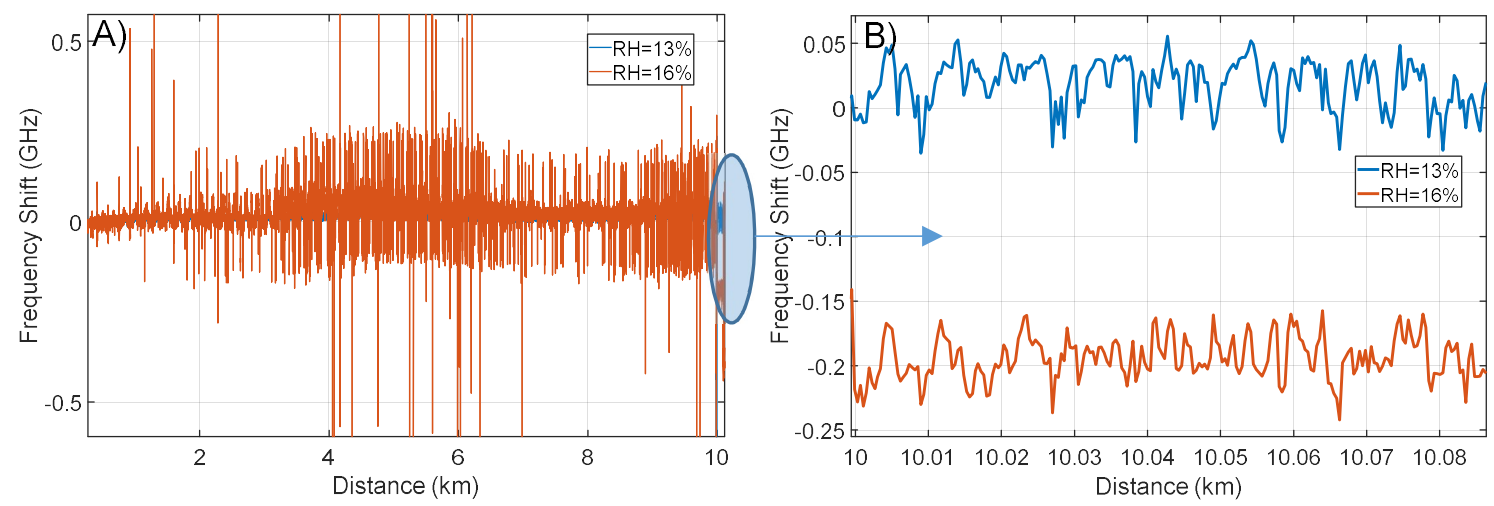

Figure 4. A) $\phi$-OTDR trace with the sensing fibre placed at the end. B) Enlarged view of the test sample section 


\section{CONCLUSIONS}

This paper shows the potential and the results of a kilometre range relative humidity sensors based on a phase-OTDR setup. The developed system is able to monitor fibres up to tens of kilometre with 70 centimetres of spatial resolution. From the measurements, it is possible to conclude that polyimide coated fibres show in general a higher sensitivity to RH then the standard acrylate fibre and stacking coating layers increases the sensitivity to RH. However, the difference between 3 and 4 layers, in the RISE fibres, is marginal. The FiberCore fibres tested show an excellent behaviour in terms of stability and sensitivity, but show a slower response ( $+45 \mathrm{mins})$ that the RISE fibres with 1 or 2 coating layers. On the other hand, no hysteresis was noticed for the FiberCore fibres, while the RISE fibres showed some hysteresis. This demonstrates that a large variety of polyimide coatings is present in the offered fibres. The Dätwyler acrylate fibre is the fastest to respond and to stabilize, but the sensitivity changes significantly with the temperature. The observed difference is around $50 \%$ passing from $25^{\circ} \mathrm{C}$ to $42^{\circ} \mathrm{C}$. The pure silica fibre from Nufren is slower ( +2 hours) and only stabilizes at $42^{\circ} \mathrm{C}$. At $25^{\circ} \mathrm{C}$, it was not possible to achieve stabilization after reasonable waiting times. The measurement resolution is slightly different from fibre to fibre but, generally, the system is able to resolve $0.1 \% \mathrm{RH}$. Due to the impossibility to change the humidity less then $1 \% \mathrm{RH}$, the resolutions is estimated from the standard deviation. If the humidity variation is higher than the double of the standard deviation, it is assumed that this system is able to resolve it. The test with the spool of $10 \mathrm{~km}$ of fibre proved that the system was able to detect a change in RH sensed by the coated fibre without any appreciable loss of sensitivity. The main conclusion of this study is that all fibres tested are good candidates to use in a distributed RH sensor. If the requirements are a fast time response and stabilization time at room temperature, the standard acrylate fibres are the best candidates, but if the requirements are higher sensitivity and measurement stability at different temperatures, polyimide fibres have advantages in several aspects.

Acknowledgments: T. Neves acknowledges M. Medeiros for the support in the reference setup development. L. Zhang and K. Hey Tow have performed this work in the framework of ITN-FINESSE, funded by the European Union's Horizon 2020 research and innovation program under the Marie Sklodowska-Curie Action grant agreement $\mathrm{n}^{\circ} 722509$.

\section{REFERENCES}

[1] F. Berghmans and A. Gusarov, "Fiber Bragg Frating Sensors in Nuclear Environments," in Fiber Bragg Grating Sensors: Recent Advancements, Industrial Applications and Market Exploitation, Benthan Books, 2011, pp. 218237.

[2] L. Alwis, T. Sun and K. Grattan, "Optical fibre-based sensor technology for humidity and moisture measurement: Review of recent progress," Measurement, vol. 46, no. 10, pp. 4052-4074, 2013.

[3] G. Berruti, M. Consales, M. Giordano, L. Sansone, P. Petagna, S. Buontempo, G. Breglio and A. Cusano, "Radiation hard humidity sensors for high energy physics applications using polyimide-coated fiber Bragg gratings sensors," Sensors and Actuators B: Chemical, vol. 177, 2012.

[4] G. Berruti, P. Petagna, S. Buontempo, A. Makovec, Z. Szillasi, N. Beni, M. Consales and A. Cusano, "One year of FBG-based thermo-hygrometers in operation in the CMS experiment at CERN," Journal of Instrumentation, vol. 11, no. 3, 2016.

[5] M. Consales, G. Berruti, A. Borriello, M. Giordano, S. Buontempo, G. Breglio, A. Makovec, P. Petagna and A. Cusano, "Nanoscale TiO2-coated LPGs as radiation-tolerant humidity sensors for high-energy physics applications," Optical Letters , vol. 39, no. 14, pp. 4128-4131, 2014.

[6] G. Berruti, T. Neves, M. Consales, P. Vaiano, G. Quero, P. Petagna and A. Cusano, "Radiation Sensitivity of Long Period Gratings written in B-Ge doped fiber under proton irradiation at CERN," in Optical Fiber Sensors 26, Lausanne, 2018.

[7] L. Thévenaz, Advanced Fiber Optics: Concepts and Tecnhology, Lausanne: EPFL Press, 2011.

[8] P. Thomas and J. Hellevang, "A fully distributed fibre optic sensor for relative humidity measurements," Sensors and Actuators B: Chemical, vol. 247, pp. 284-289, 2017.

[9] X. Lu, "Coherent Rayleigh time domain reflectrometry: novel application for optical fibre sensing," EPFL PhD thesis 7104, 2016.

[10] L. Zhang, L. Costa, Z. Yang, M. Soto Hernandez, M. Gonzalez-Herráez and L. Thévenaz, "Analysis and Reduction of Large Errors in Rayleigh-based Distributed Sensor," Journal of Lightwave Technology, 2019. 